\title{
Numerical Simulation of Induction Heating of Steel Plate Products
}

\author{
Victor Demidovich \\ Faculty of Electrical Engineering \\ St.Petersburg Electrotechnical University (LETI) \\ St.Petersburg, Russia \\ vbdemidovich@mail.ru
}

\author{
Yuri Perevalov \\ Faculty of Electrical Engineering \\ St.Petersburg Electrotechnical University (LETI) \\ St.Petersburg, Russia \\ yyperevalov@yandex.ru
}

\author{
Olga Demidovich \\ Faculty of Electrical Engineering \\ St.Petersburg Electrotechnical University (LETI) \\ St.Petersburg, Russia \\ ovdemidovich@mail.ru
}

\begin{abstract}
Main methods of induction heating of steel plate products are discussed in this paper. Different 2D and 3D models are developed for investigation and design of the induction coils for heating steel slabs and strip. A new concept of universal induction coils that allow using a combination of longitudinal and transverse flux for heating plate products is discussed in this paper.
\end{abstract}

Keywords - Coupled electromagnetic and temperature fields, electromagnetic processing of metals, induction heating, multiphysics problems

\section{INTRODUCTION}

The induction heating method can be used in steel industry for heating slabs and strip. Historically, hot-rolling mill operators in the steel industry have not widely utilized induction heating due to the belief that induction heaters have high energy and capital costs and that the technology is not well understood. Induction heaters, however, offer rapid heating, precise temperature control, and consistent properties that are desired by rolling mill operators and often justify any associated higher costs.

Only a few induction furnaces are operating now for heating thick slabs with 150-250 mm thickness. Nevertheless they show high reliability and high quality of the product.

More often, induction heaters are used for reheating thin slabs with $25-50 \mathrm{~mm}$ thickness between the roughing and the finishing mills. It may be necessary to reheat the transfer slab between the two rolling stages, depending on the casting conditions and on the final product. An induction furnace, which can be switched on and off as required, is found to be an economical instrument for such flexible intermediate heating.

Another important application of the induction heating of the plate products in steel industry is heating of strip. The first investigations and publications about the industrial application of induction heating for steel strip were carried out in the early 1940s $[1,2]$. However, widespread industrial application of the induction strip heating did not occur until the 1960s [3,4], that is, when the steel industry first implemented continuous heat treating of the strip, replacing the older batch heat treating technology.

There were already a number of ideal applications for induction heating for steel strip. Some of these were partial and full annealing, normalizing, tempering, applying different types of strip coatings, and paint drying.

In general, there are two main strategies used for induction heating of the plate products - heating in the magnetic longitudinal or in the magnetic transverse fluxes. In the longitudinal flux method, induction coils are arranged in such a way that the coils' currents generate a magnetic flux primarily in the direction parallel to the plate surface. On the other hand, the transverse flux concept positions the coils so that their currents generate a magnetic flux that travels mainly through the flat surface of the metal in the direction perpendicular to the plate surface. In the longitudinal heating case, induced eddy currents circulate within the strip thickness. An acceptable electric efficiency can be achieved in this case if the thickness of the plate, d, is at least twice penetration depth of electromagnetic wave in the metal. For the transverse magnetic flux heating, it is preferable to have a penetration depth of more than the thickness of the plate. The induced eddy currents are then circulated in the plane of the plate, which means that for heating of a plate with the same thickness, it is possible to use cheaper power source with much lower frequency.

\section{INDUCTION HEATING OF STEEL STRIP IN LONGITUDIAL FLUX}

Longitudinal Flux Heating (LFH) requires a particular design for its induction coils. A simple solenoid coil surrounding the plate is generally used for this concept. The width and opening of the coil depend on the maximum width of the strip. Usually, the opening of the coil is relatively large, to prevent contact between the plate and the structure. For some technologies, it is necessary to move the coils away from 
line without disturbing the steel plate. A special coil design with the concept of the longitudinal flux is used for steel strip heating in steel strip galvanizing application [5]

The electrical efficiency of an induction coil for steel strip heating in a longitudinal magnetic field strongly depends on the frequency, the thickness of the strip, the specific power density, and the temperature of the strip at the entrance and the exit of the inductor. The thermal efficiency does not depend as strongly on these parameters as electrical efficiency does, but must still be taken into account. In further analyses, a common efficiency is used. The common efficiency is defined as the product of the electrical and thermal efficiencies. One of the main design criteria of induction coils for heating ferrous steel strip is to achieve a common efficiency of no less than $90 \%$. Therefore, the choice of the proper frequency is very important in design of the induction coil.

Fig. 1 shows the minimum frequency required for the induction heating of a low carbon steel strip with different thickness and strip exit temperatures, which guarantee high coil efficiency. In each case, the strip is heated from room temperature. Higher strip exit temperatures from the inductor require the higher frequencies (Fig. 1). The need to increase the frequency with increase in exit temperature of the strip is especially pronounced when the strip temperature is close to

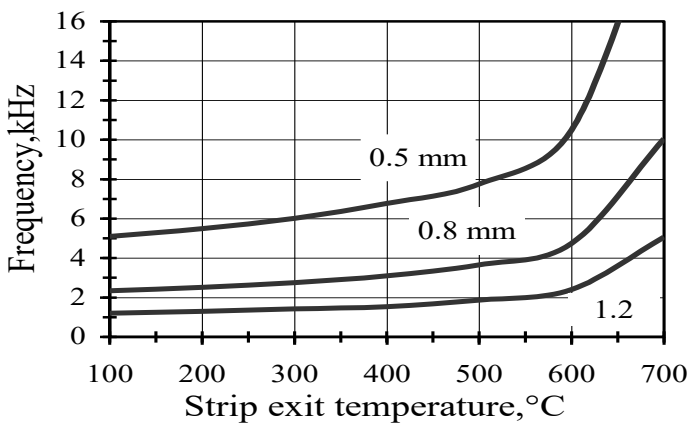

Fig. 1. Lowest level of frequency to have 0.9 efficiency. Strip entrance temperature is $20^{\circ} \mathrm{C}$. Specific power is $40 \mathrm{~W} /(\mathrm{cm} \cdot \mathrm{cm})$.

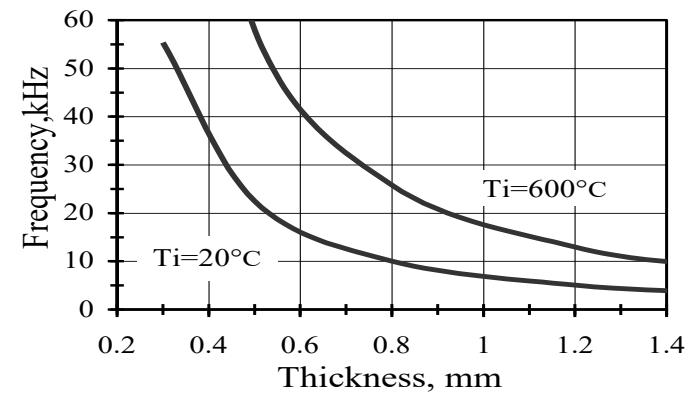

Fig. 2. Lowest level of frequency to have 0.9 efficiency. Strip exit temperature is $700^{\circ} \mathrm{C}$. Specific power is $40 \mathrm{~W} /(\mathrm{cm} \cdot \mathrm{cm})$. the Curie temperature $\left(725^{\circ} \mathrm{C}-750^{\circ} \mathrm{C}\right)$. The required frequency dramatically increases when it is necessary to heat strip above $600^{\circ} \mathrm{C}$ for all gauges. Low temperature heating requires relatively low frequencies and inexpensive power sources.

Fig. 2 shows how the thickness of the carbon steel strip impacts the choice of frequency. The figure shows that for a thinner strip, it is necessary to choose a higher frequency. It is important to note that higher strip entrance temperatures also require higher frequencies. For example, if the strip entrance temperature is $600^{\circ} \mathrm{C}$, the minimum necessary frequency is approximately double the frequency required when the strip entrance temperature is $20^{\circ} \mathrm{C}$.

Specific power density also affects the choice of the frequency (Fig. 3). Decreasing the specific power for low temperature strip heating (below $300^{\circ} \mathrm{C}$ ) allows the use of a lower frequency. However, for the high temperature strip heating $\left(700^{\circ} \mathrm{C}\right)$, the opposite is true. It then becomes necessary to use a higher frequency because thermal losses must be taken into account, especially at a low specific power density of the induction heating.

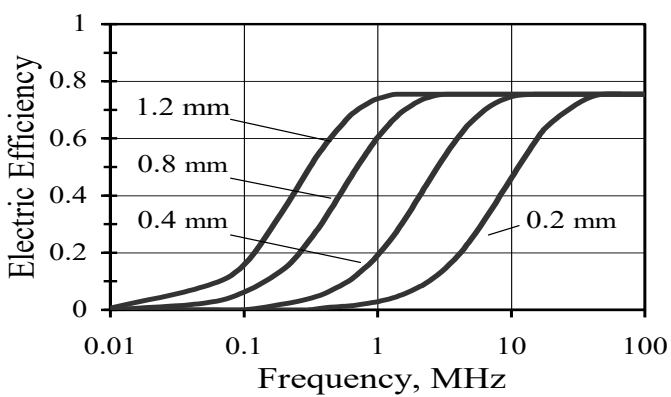

Fig. 4. Electric efficiency of non-ferrous steel strip heating in longitudinal magnetic field.

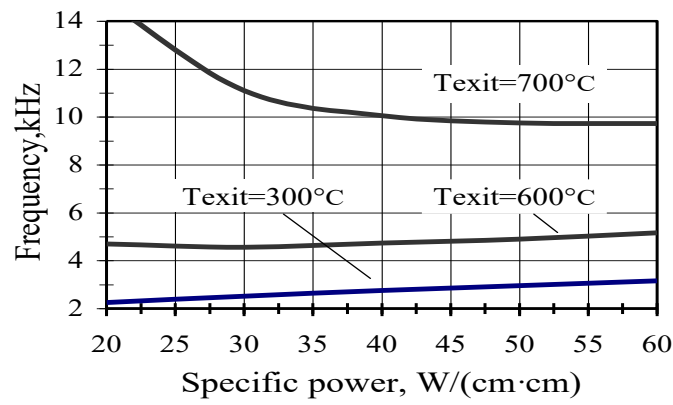

Fig. 3. Lowest level of frequency to have 0.9 efficiency. Thickness of the strip is $0.8 \mathrm{~mm}$. Strip entrance temperature is $20^{\circ} \mathrm{C}$. 
A significant advantage of induction heating with a longitudinal flux is a good homogeneity of the temperature across the width of the strip. The relatively long solenoid induction coil induces a uniform longitudinal magnetic field along the perimeter of the strip cross section. Consequently, only a small area at the edge of the strip could be overheated or under-heated. The temperature difference between the edge and the main part of the strip does not exceed $8-10^{\circ} \mathrm{C}$ for strips of any thickness.

For the induction heating of a non-ferrous steel strip, it becomes necessary to use much higher frequency (Fig. 4). Electric efficiency in this case will not exceed $75 \%$. Taking into account the thermal efficiency, which could be sufficiently great with the temperature of the strip above the Curie temperature, the resulting common efficiency will be much lower than it would be for the heating of a ferrous strip. Therefore, in non-ferrous thin strip heating situations, transverse magnetic flux should be used.

\section{INDUCTION HEATING OF STEEL STRIP IN TRANSVERSE FLUX}

Transverse flux heating has limited usefulness for steel strip processing because of several reasons. Heating of carbon steel strip below the Curie temperature can be realized with a very high electrical efficiency (more than 90\%) using longitudinal flux inductors. The implementation of Transverse Flux Heating (TFH) does not increase the electric efficiency. Modern transistorized generators with a current frequency close to $1 \mathrm{MHz}$ allows longitudinal flux heating in the very thin ferrous strip. In the case of longitudinal flux heating of a ferrous strip, it is possible to avoid the problems associated with TFH systems, such as a non-uniform temperature distribution across the width of the strip, thermal deformation of the thin strip and electromagnetic forces on the strip.

The induction heating with transverse flux can be adopted only for processing stainless steel or carbon steel strip and thin slabs at temperatures above the Curie temperature. In this case, transverse flux heating has the advantage over longitudinal flux heating in terms of electrical efficiency.

The choice of frequency for induction heaters using transverse magnetic flux is more complicated than for installations with longitudinal flux. The complications arise from the fact that the frequency depends not only on the properties and thickness of the strip, but also on the design of

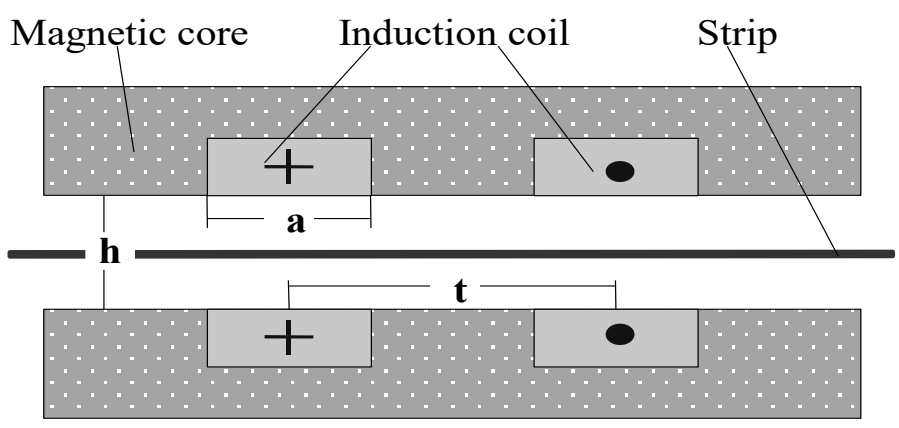

Fig. 5. Cross section of the inductor with magnetic transverse flux. the induction coils. The cross section of the typical inductor design for transverse flux heating is shown in Fig. 5.

The height of the air gap, h, (opening) more strongly impacts the design of the induction coil in transverse flux heating scenarios than when longitudinal flux heating is used. Usually induction coils heating with a longitudinal flux have a length of at least 5 times the height of the air gap. Fluctuations in the location of the strip relative to the coil do not influence the power transfer to the strip, electric efficiency of the coil, power factor of the coil, and the impedance of the coil. A first guess at the pole pitch, $t$, and width of the slot, a, should be made to provide the minimum deviation in the power transfer to the strip when the distance between the strip and the top and bottom inductors varies. This effect can not be evaluated from the R. Baker theory [2]. In the early 1960s, V. Peysakovich used a 2D model to recommend guidelines for choosing appropriate values for $t$ and a for a prescribed air gap height, $h$ [6]:

$$
\begin{array}{ll}
\mathrm{h} / \mathrm{t}<0.75 & \text { if } \mathrm{a} / \mathrm{t}<0.25 \\
\mathrm{~h} / \mathrm{t}<0.5 & \text { if } \mathrm{a} / \mathrm{t}<0.75
\end{array}
$$

This recommendation can be used as a general guideline, but should be checked using a $3 \mathrm{D}$ model. It is also important that the pole pitch, $t$, should be larger than the minimum strip

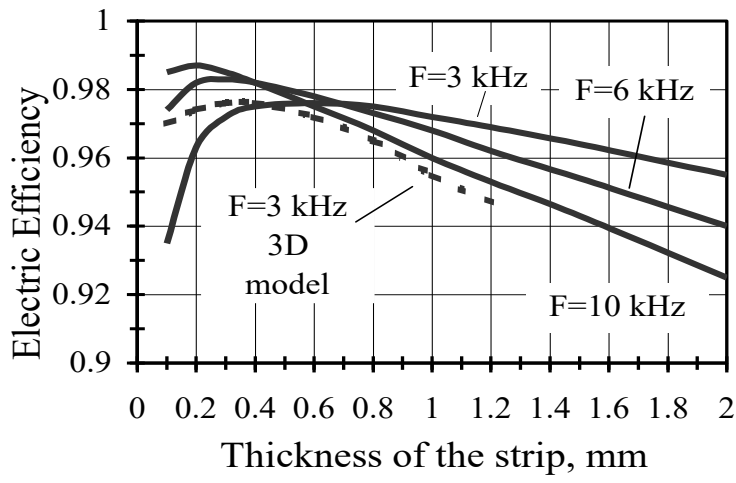

Fig. 6. Electric efficiency of TFH steel strip with temperature above Curie $(\mathrm{a}=200 \mathrm{~mm}, \mathrm{t}=300 \mathrm{~mm}, \mathrm{~h}=140 \mathrm{~mm})$.

width, b. Analyses have shown that the maximum electric efficiency is achieved if $0.5<\mathrm{a} / \mathrm{t}<0.75$.

Generally, the selection of a frequency was made such that maximum electric efficiency and power factor were attained. Fig. 6 shows how electric efficiency depends on the thickness of the steel strip at temperatures above the Curie temperature and at different frequencies. The computations are performed using a 2D model. In the specific instance of a $10 \mathrm{kHz}$ frequency, a 3D model was also used. There is no clear maximum attainable efficiency to dictate the optimal frequency. For that reason, the frequency may be chosen from a wide range. Often, the recommended frequency is chosen on the basis of maximizing the power factor (Fig. 6). However, the main problem that prevents the widespread 
implementation of transverse flux heating is the sensitivity of the power distribution (and therefore temperature distribution) across of the strip width to changes in the relative positioning of end parts of the inductor and the edge of the strip $[7,8]$.

\section{NEW CONCEPT OF INDUCTION COILS FOR HEATING STEEL STRIP AND THIN SLABS}

Depending on the heat-treating cycle being implemented, the annealing temperature of low carbon steel strip in a galvanizing line could be above or below Curie temperature [9]. Using two different types of induction coils (coils using longitudinal flux below Curie temperature and coils using transverse flux above Curie temperature) is inconvenient and expensive. Instead, a different approach can be taken.

The principle design of the new universal induction coils for heating a strip and thin slabs [10] can be made based on using coils for transverse flux heating. The top and bottom coils are connected to the power source that has capability to change the phase angle between the currents of the coils. The phase angle is $0^{\circ}$ when configured for transverse flux and $180^{\circ}$ - when configured for longitudinal flux. A mixed magnetic flux for heating could be received if the shifts of current phase angles are between $0^{\circ}$ and $180^{\circ}$.

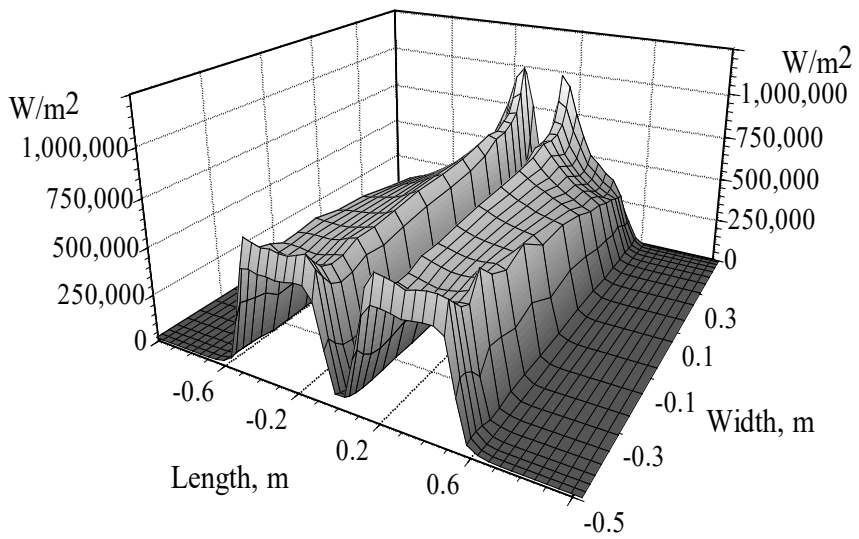

Fig. 7. Power distribution, phase shift of $180^{\circ}$

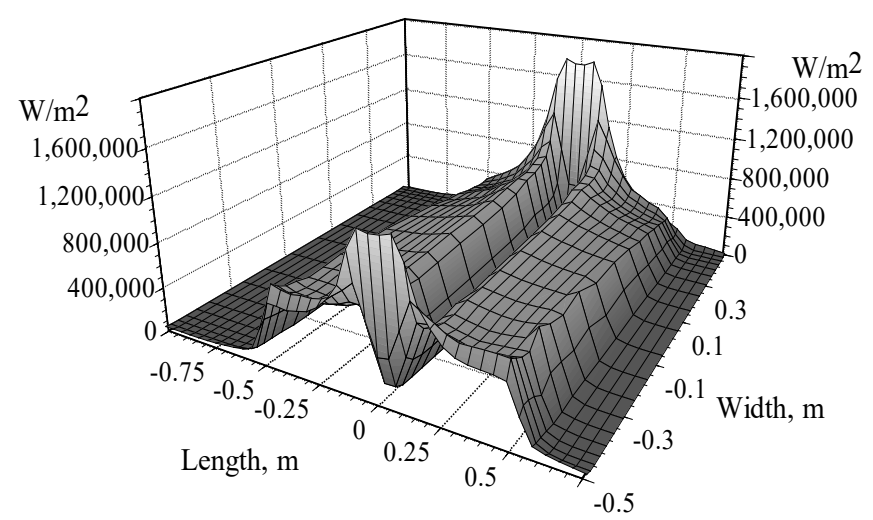

Fig. 8. Power distribution, phase shift of $165^{\circ}$

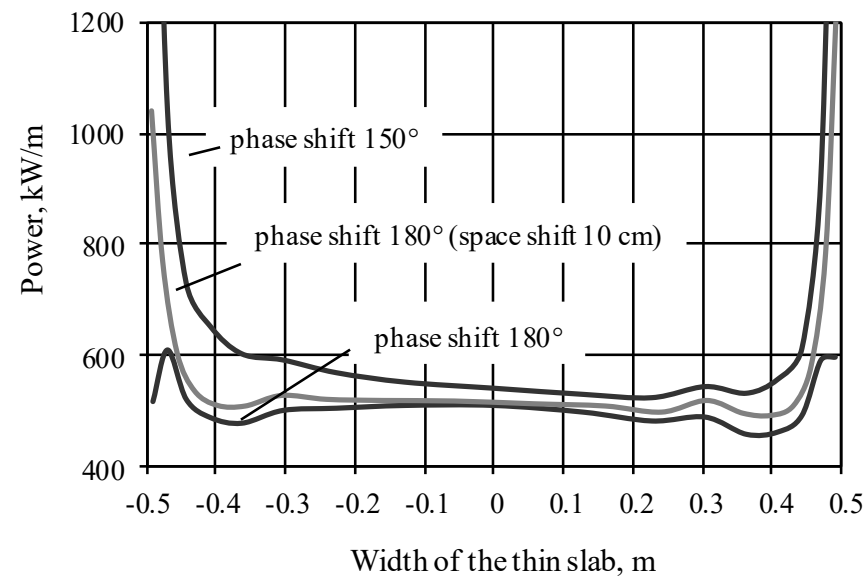

Fig. 11. Power distribution across the width of the thin slab

If the power source can not smoothly change current phase angles for the induction coils, it is possible to arrange simple switching of the phase from $180^{\circ}$ to $0^{\circ}$, i.e. from longitudinal flux to transverse flux in the same induction system. Space shift between the top and bottom coils along the length of the plate products allows redistributing power across the width, for example, of the thin slap.

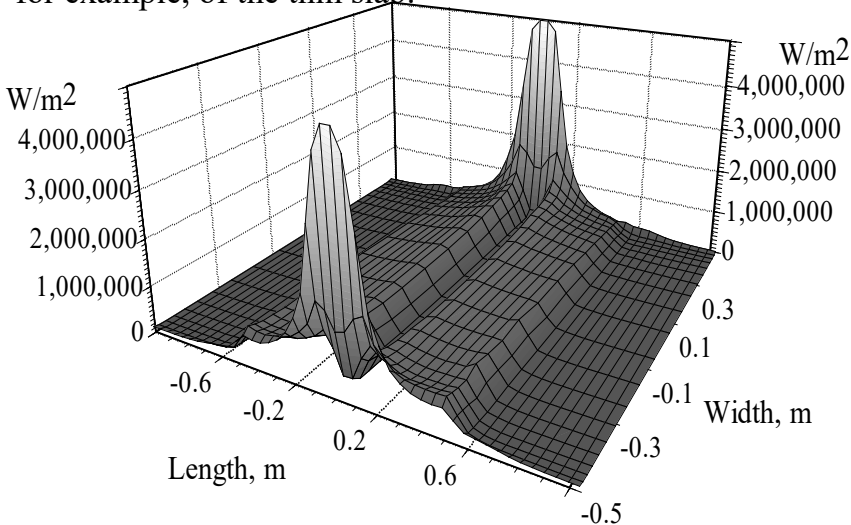

Fig. 9. Power distribution, phase shift of $150^{\circ}$.

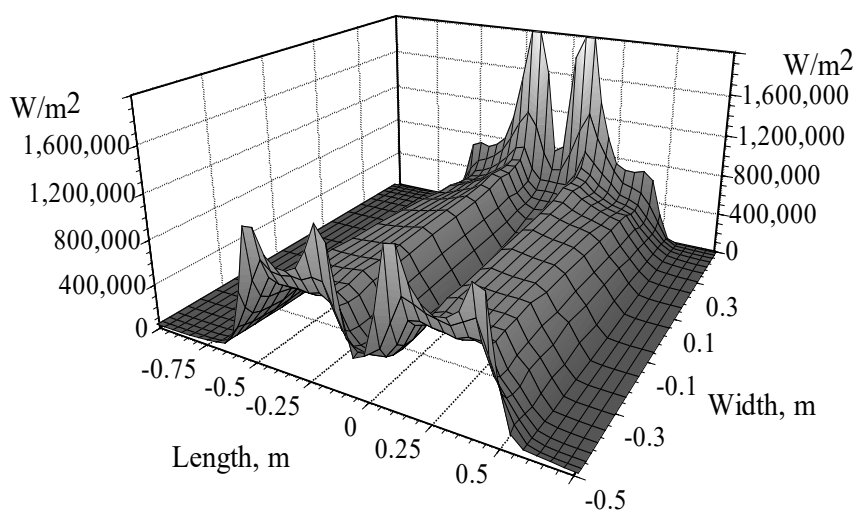

Fig. 10. Power distribution, space shift of $10 \mathrm{~cm}$. 


\section{Example. Induction reheating of thin slab with average initial temperatures of $900^{\circ} \mathrm{C}$}

The thickness of the thin slab is $25 \mathrm{~mm}$, width is 1 meter. Parameters of the inductor's coils: air gap is $\mathbf{h}=165 \mathrm{~mm}$, width of the slot is $\mathbf{a}=400 \mathrm{~mm}$, pole pitch is $\mathbf{t}=600 \mathrm{~mm}$. Frequency is $3000 \mathrm{~Hz}$. Power distributions in the thin slab are shown in Fig. 7 - 10. Fig. 7 - 9 show the power distribution in the slab with different phase shifts between currents from the top and bottom coils. Fig. 10 shows the power distribution when the phase shift is $180^{\circ}$ (longitudinal flux), but there is a $100 \mathrm{~mm}$ space shift between the top and bottom coils. The electric efficiency in all cases is between $65 \%$ and $70 \%$. The power distribution across the width of the thin slab for all these cases is shown in Fig. 11. The flexibility to change the phase angle between currents allows combining the reheating and slab edge heating furnaces into one unit.

\section{MODELING OF INDUCTION STRIP HEATING}

For the design of an induction strip heater, several 1D, 2D, and 3D electromagnetic and coupled electromagnetic-thermal models have been developed $[4,7,8]$. In the case of ferrous strip heating, some special modeling features were taken into account because of the non-linear nature of the system.

Codes for the simulation of induction heating should provide the capability to solve the tightly coupled (interrelated) computations of electromagnetic and heat transfer phenomena. In order to create this feature, a special computational algorithm has been developed [4]. This algorithm is based on jointly solving a system of two nonlinear differential equations, which describe the electromagnetic and temperature fields, and provides a reliable coupling of both phenomena.

In order to achieve the desired accuracy and efficiency of the computations, the program system was developed to model induction strip heating. It consists of three groups of models.

\section{A. Models for induction coils with longitudinal flux}

Special algorithm was developed that comprised two 2D models. The first one allows receiving distribution of electromagnetic and temperature fields in the each crosssection of the strip and billets along the length. This model is based on the FEM in the Ritz approach. The second model allows predicting distribution of the electromagnetic field along the length of the strip.

\section{B. Models for induction coils with transverse flux}

For this particular case, two models were developed. The first one is based on the 2-D analytical solution [6] of the electromagnetic problem in the cross section of the TFH induction system. It allows the user to make a first guess of the frequency and main design parameters of the TFH system. The other model was developed on the basis of the combining method [4], which is comprised of the utilization of the Integral Volume Method (IVM) for computation of the external Electro Magnetic Field (EMF) outside the strip and FEM or FED for computation of the EMF inside of the strip

\section{Model for universal induction coils}

In addition to the above-mentioned models, the commercial package, ANSYS, was used to investigate the 3-D electromagnetic fields and to verify the models.

\section{CONCLUSION}

Different induction coil designs can be used to heat steel strip. The final decision is based on overall efficiency, cost of installation, elimination of the possible heating of the surrounding structural elements, exclusion of the EMF radiation to the people at the service area, easy removal of the coils without disturbing the strip, and the ability to heat the strip at temperatures exceeding the Curie temperature.

Using developed models of induction strip heaters makes it easy to compare the different options.

For steel strip heating to temperatures above the Curie temperature, it is possible to use the same inductors and the same power sources by heating the strip with longitudinal magnetic flux below the Curie temperature and with transverse magnetic flux above Curie temperature.

This design of induction coils can also be used for reheating and edge heating of thin slabs.

\section{ACKNOWLEDGMENT}

The work was carried out with the financial support from the Ministry of Education and Science of Russia in the framework of the state work "Organization of Scientific Research" (assignment No.8.6037.2017 / VU).

\section{REFERENCES}

[1] V.P. Vologdin, A. E. Slukhotsky, Device for Heating Metal Shits, USSR Patent 60670, 1942

[2] R. Baker, "Transverse Flux Induction Heating", Electrical Engineering (AIEE Transactions), vol. 69, pp. 711-719, 1950.

[3] M.G. Lozinskii, "Industrial Application of Induction Heating", Pergamon Press, London, 1969.

[4] V.S. Nemkov, V.B. Demidovitch, "Theory and Computation of Induction Heating Devices", Energoatomizdat, Leningrad, 1988

[5] H. Rowan, J. Mortimer, D. Loveless, "Continuous Strip Material Induction Heating Coil”, United States Patent 5,495,094, 1996.

[6] V.A. Peysakovich, "Problem of uniform heating of moving metal strip in transverse magnetic flux", Industrial application of HF currents, M.L., Mashgis, vol. 53, 1961.

[7] Muehlbauer, A. Ruhnke, V.B. Demidovitch, A.N. Nikanorov, S. Lupi, F. Dughiero, "Methods and Tools for All-Round Optimization of Transverse-Flux induction Heaters", in Proceedings 1997 International Induction Heat Treating Symposium, Indianapolis, USA, pp. 865-870.

[8] E. Mannanov, S. Galunin, K. Blinov, Numerical Optimization of Transverse Flux Induction Heating Systems Proceedings of the 2015 IEEE North West Russia Section Young Researchers in Electrical and Electronic Engineering Conference, ElConRusNW 2015, 2015, pp. 241-244.

[9] N. Ross, R. Scherer, D. Jancosek, "Induction Heating of Strip for Galvanneal", Iron and Steel Engineer, Vol. 40, 1988.

[10] V.B. Demidovitch, J. Nelson, E. Blake, "New Generation of Galvanizing Lines Utilizing Induction Heating Technology", Proceedings 2000 EPM2000, Nagoya, Japan, pp. 224-230. 